\title{
A User Interface for Query-by-Sketch based Image Retrieval with Color Sketches
}

\author{
Ivan Giangreco, Michael Springmann, Ihab Al Kabary, and Heiko Schuldt \\ Databases and Information Systems Research Group \\ Department of Mathematics and Computer Science, University of Basel, Switzerland \\ firstname.lastname@unibas.ch
}

\begin{abstract}
This demo will interactively show a system that exploits a novel user interface, running on Tablet PCs or graphic tablets, that provides query-by-sketch based image retrieval using color sketches. The system uses Angular Radial Partitioning (ARP) for the edge information in the sketches and color moments in the CIELAB space, combined with a distance metric that is robust to deviations in color as they usually need to be taken into account with user-generated color sketches.
\end{abstract}

\section{Introduction}

Over the last few years, the sizes of image archives have been rapidly growing which makes querying for specific images, also referred to as known item search, increasingly difficult. As more and more images are not annotated with proper metadata, content-based image retrieval (CBIR) is a viable approach to image retrieval that solely relies on the inherent visual characteristics. A major problem in CBIR is however the availability of a query image that is good enough to express the user's information need. Query-by-sketch allows to overcome this limitations as users can contribute sketches as query input. In contrast to most existing approaches that consider only edge information extracted from the sketch, this demo presents a system that jointly exploits edge and color information, gathered via a novel, user-friendly interface.

\section{The Demo}

In this demo, we present an approach based on the QbS system [3] that seamlessly combines a color descriptor using the perceptually uniform CIELAB color space with the Angular Radial Partitioning (ARP) [1] for CBIR based on userprovided color sketches. For the regions defined by ARP, we use the first two color moments and the joint moment as features and apply a weighted, $\varepsilon$-insensitive $L_{1}$-distance for a k-Nearest-Neighbor $(\mathrm{kNN})$ search. A distance combining function and multiple distance normalization functions are applied to jointly consider color and edge features. The QbS system has been subject to an evaluation based on the MIRFLICKR-25'000 image collection [2] that shows that the introduced color descriptor generally produces better results than the edge-based descriptor. Combining both descriptors in almost all cases significantly improves the retrieval when using handwritten sketches. 


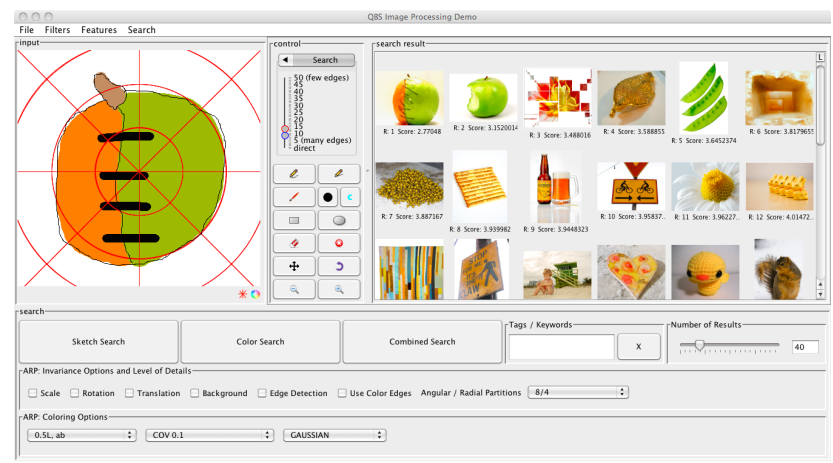

Fig. 1. Sample query based on a user-provided, colored sketch.

Figure 1 shows the QbS interface, together with the retrieval results that have been obtained for a search using color and edge information (right hand side). On the input area (left hand side), the ARP regions are displayed as an overlay on the sketch, to illustrate the partitions which are used for image comparison.

In order to make color selection and the coloring of an edge sketch as userfriendly as possible, a set of tools is made available in the query frontend as marking menus, a special form of pie menus. With such marking menus, the user does not need to lift the pen or finish the click to choose an element, which makes them highly appropriate for the task of colorizing sketches. The combination of edge and color search necessitates a sophisticated organization of the sketching canvas. For this, QbS uses a multi-layered canvas, transparent to the user, which is responsible for separating the sketches drawn for edge search from the colorized areas for color search. In combination with a separate layer for the background this allows for an optional separate treatment of sketching and coloring. The latter is beneficial to assess the retrieval quality of the combined approach with regard to edge-only and color-only search, respectively.

Acknowledgements. This work was partly supported by the Swiss National Science Foundation (project PAD-IR).

\section{References}

[1] Chalechale, A., Mertins, A., Naghdy, G.: Edge Image Description Using Angular Radial Partitioning. IEE Proceedings Vision, Image \& Signal Processing 151(2), 93-101 (Apr 2004)

[2] Huiskes, M., Lew, M.: The MIR Flickr Retrieval Evaluation. In: Proceedings of the 1st ACM International Conference on Multimedia Information Retrieval (MIR 2008). pp. 39-43. ACM, New York, USA (2008)

[3] Springmann, M., Al Kabary, I., Schuldt, H.: Image Retrieval at Memory's Edge: Known Image Search based on User-Drawn Sketches. In: Proceedings of the 19th ACM International Conference on Information and Knowledge Management (CIKM 2010). pp. 1465-1468. ACM, New York, USA (Oct 2010) 